\title{
THE BEHAVIOUR OF VIRTUAL SYNCHRONOUS MACHINE (VSM) BASED CONVERTERS IN FRONT OF NON-SATURABLE FAULTS
}

\author{
Zhenkun Yang $^{*}$, Agustí Egea-Àlvarez ${ }^{2}$, Adam Dysko ${ }^{3}$ \\ ${ }^{l}$ Electronic and electrical engineering department, University of Strathclyde, Glasgow, U.K. \\ *zhenkun.yang@strath.ac.uk \\ agusti.egea@strath.ac.uk \\ a.dysko@strath.ac.uk
}

\begin{abstract}
Keywords: VIRTUAL SYNCHRONOUS MACHINE, FREQUENCY STABILITY, INERTIA RESPONSE, CURRENT VECTOR CONTROL, POWER SYNCHRONISATION CONTROL
\end{abstract}

\begin{abstract}
Power converter penetration has increased substantially in the last 20 years bringing new challenges from the system protection perspective. The power network is undergoing a major transformation as the major part of new installed power comes from nonsynchronous sources such as wind or solar. These changes might lead to malfunction of the conventional protection schemes such as overcurrent protection or distance protection relays. At the same time, the reduction of the system inertia might cause the tripping of the Loss of Main protection due to a very aggressive Rate of Change of Frequency. To enhance the grid voltage source characteristic and mitigate the loss of inertia, a new set of converter controllers known as Grid forming Converter or Virtual Synchronous Machine has been suggested in recent years. The performance of VSM could provide a potential advantage compared to traditional power converter controllers when a large frequency deviation occurs helping to keep the system stable. This article quantifies and compares the performance of different converter control algorithms including Current Vector Control, Virtual Synchronous Machine and Power Synchronisation Control in front of different frequency events.
\end{abstract}

\section{Introduction}

With the growing penetration of renewable energy generation connected to the power system, conventional protection methods are facing a challenge due to the power converter reduced contribution to fault level [1][2]. Previously, the design philosophy of power system protection is based on the assumption that power is mainly generated by the synchronous generator (SG), which performs as a voltage source and is able to provide a large amount of fault current during the disturbance. However, in the network with high penetration of converters, the converters will behave like a current source to protect themselves. In addition, the fault current will be lower $(1-1.5$ p.u. $)$ due to the lack of thermal inertia of the converter switches and a short time delay might exist [3].

This may cause two problems from the perspective of system protection. First, the lack of voltage-source characteristic may result in the mal-operation of conventional protection devices such as over current and distance relays, the protection of which can be under-reached in networks with high-penetration of power converters [4][5]. Second, frequency stability issues caused by the nature of low-inertia in the future network challenges the conventional protection scheme. The prevalent Loss of Main protection (LOM) based on the detection of Rate of Change of Frequency (ROCOF) might be activated for high ROCOF values. This event might start an undesired cascade relay-tripping event that might lead to a black out. [5]

The proposed method of Virtual Synchronous Machine (VSM) is a potential way to enhance the voltage-source characteristic of the future network and solve the frequency stability problems. VSM controllers mimic the behaviour of a SG in different degrees of detail, and show improved performance compared to conventional control strategies, under various frequency and fault events. There are several versions of VSM models developed in recent years, designed for different purposes since 2007 [6]. In some research, full-order VSM including virtual fluxes, torques, damping windings is developed to mimic the entire behaviour of real SG, however, the control algorithm can be complex. Some researchers have developed simplified VSM controllers based on the swing equation. For example, the Power Synchronisation Control (PSC) model proposed in 2011 and Virtual Synchronous Machine with Zero Inertia (VSMOH) model put forward in 2017 [7][8]. From the network protection perspective, VSM can also offer a potential solution as it can prevent the conventional relays from incorrect operation, and it can also mitigate nuisance tripping of the LOM protection caused by the system frequency events.

This paper compares the behaviour of different types of VSM implementations under the remote fault conditions where the power converter does not reach the nominal current. During the test, three types of control method are compared with each other: (1) Current Vector Control (CVC) model with inertia emulation loop; (2) VSM model; (3) PSC model. The paper is organised as follows: The detailed configurations and settings of different models are demonstrated in section 2. The simulation result and analysis are explained in section 3 . Section 4 summarizes the results and draws the more general conclusions. 


\section{Controller implementation}

In this section, the configuration of three different models are described. The compared controllers are Current Vector Control with inertia emulation (CVC), Virtual Synchronous Machine (VSM), and Power Synchronisation Control (PSC).

\subsection{Inertia emulation in Current Vector Control (CVC)}

The overall configuration of conventional CVC is shown in Figure 1. The control is implemented in the synchronous reference frame where a Phase Locked Loop (PLL) is used as grid synchronisation method. The inner current control loop controls the current through the coupling inductance and the outer controllers regulate the power and voltage at the point of connection. To enhance the converter inertia behaviour during the disturbance, the inertia emulation loop is added to adjust the power reference during the grid frequency event [9].

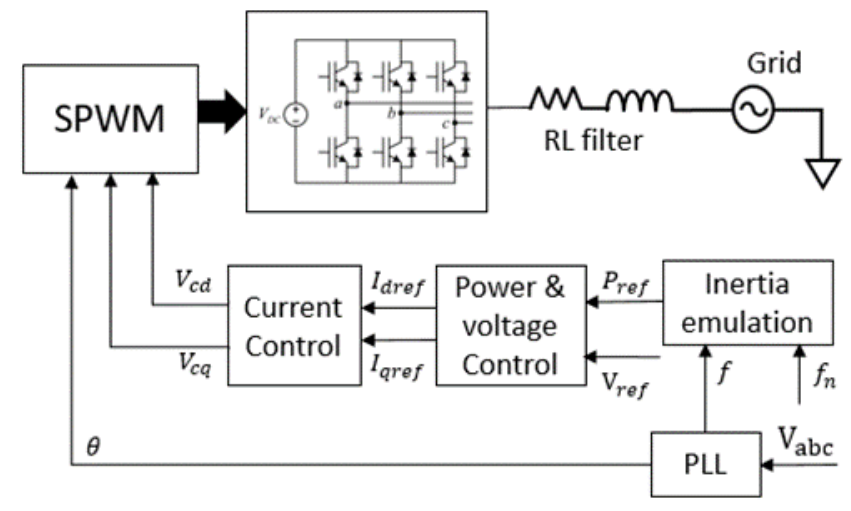

Figure 1 Current Vector Control

Figure 2 presents the sketch of the current loop where two proportional integral (PI) controllers are used to control $q$ and $d$ components of the current. The values of the gains $k_{P I}$ and $k_{I I}$ depend on the required bandwidth of current controller and filter impedance values [10].

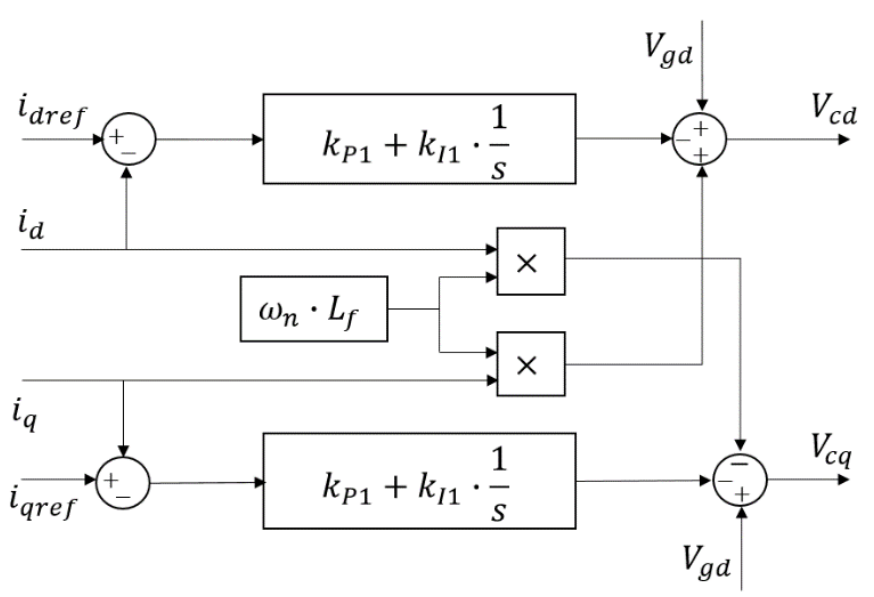

Figure 2 Current control

In Figure 3, the outer loop consists of two PI controllers that control the AC active power and the voltage at the point of connection.
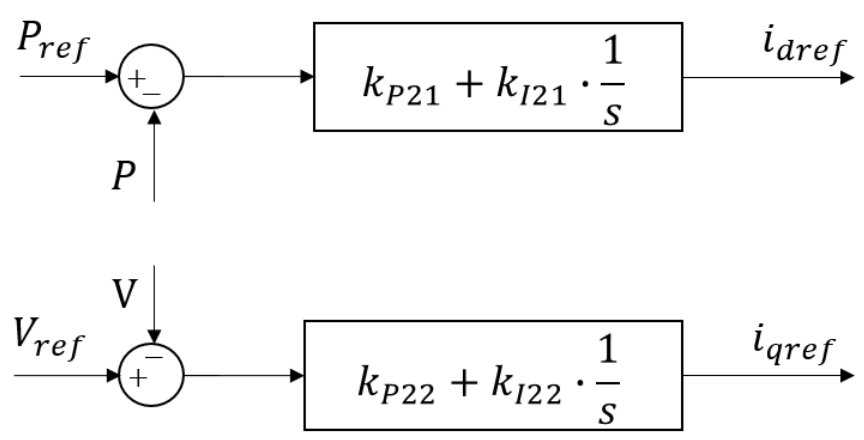

Figure 3 Power and voltage control

Figure 4 illustrates the implementation of the inertia emulation loop. The controller provides (or absorbs) active power when the measured frequency deviates from the nominal value as a SG would do. The parameter $k_{D}$ represents the inertia time constant of a SG, and is designed according to the required inertia support during the disturbance.

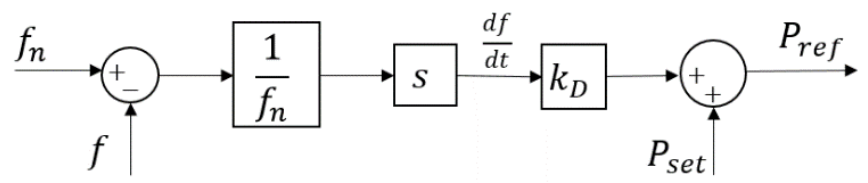

Figure 4 Inertia emulation loop

\subsection{Virtual Synchronous Machine (VSM)}

The Figure 5 shows the general structure of VSM. The voltage is controlled by a PI controller with the parameters of $k_{P 3}$ and $k_{I 3}$, similar to a Synchronous Machine voltage controller and the active power is controlled by a PI+I controller designed to emulate the swing equation. This VSM has no current vector control loop and no PLL. However, due to the lack of current control, the current cannot be limited using conventional methods during a fault [11].

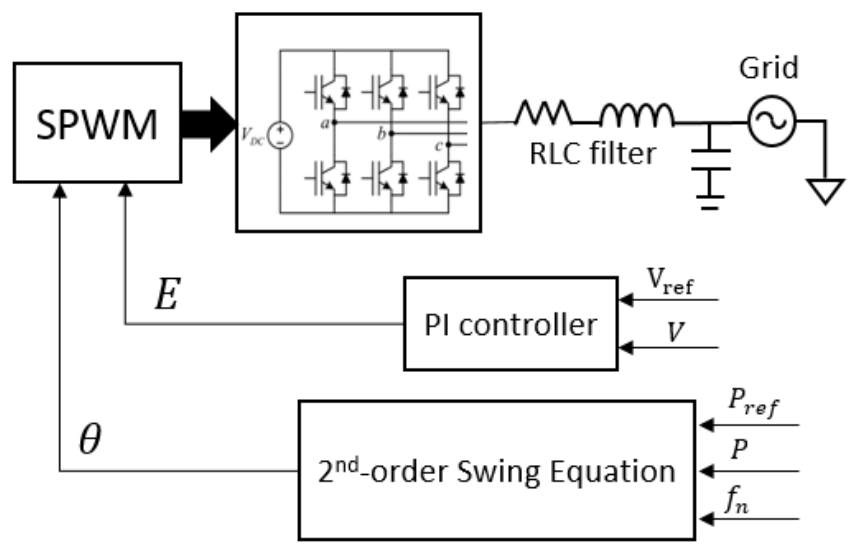

Figure 5 Virtual Synchronous Machine

The detailed structure of active power controller progress is shown in Figure 6. The power difference between reference and measured value will be used to generate the phase angle through two integration progresses. This control loop resembles to the second-order swing equation of $\mathrm{SG}$ : $\frac{2 H}{\omega_{n}} \cdot \frac{d \delta^{2}}{d t^{2}}=P_{m}-P_{e}-D \cdot\left(\omega-\omega_{n}\right)$. 
$P_{r e f}$ works as the mechanical power and $P$ works as electrical power. In addition, $k_{p}=\frac{D}{2 H \frac{1}{X} \omega}$ and $k_{I}=\frac{1}{2 H}$. $H$ represents the inertia constant related to the kinetic energy in the rotating mass of the rotor over the nominal apparent power value of SG. $D$ is the damping factor that will control the power input based on the deviation between the measured grid frequency and nominal frequency.

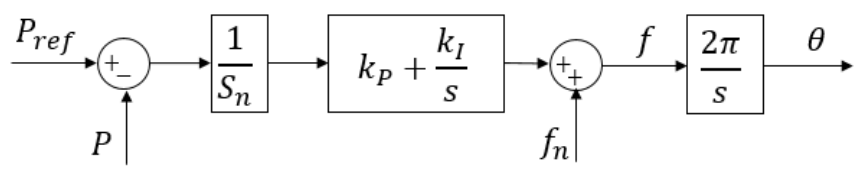

Figure 6 2nd-order power control

\subsection{Power Synchronisation Control (PSC)}

The PSC is a particular implementation of VSM that has a current loop to provide fault current during faults. The modelling of PSC is based on the performance of SG in the conventional grid; however, this method does not apply the second-order swing equation because the double integration from power to angle may lead to the significant inherent damping and poor phase stability margin [7]. Reference voltage control loop is implemented to provide the required reference for the voltage. In this way, the controller will contain both power synchronisation characteristic and current limiting capability at the same time. The configuration of PSC is demonstrated in Figure 7, and the voltage control loop is shown in Figure 8.

The PI controller parameters $k_{P 4}$ and $k_{I 4}$ of the voltage controllers have been designed to satisfy the required cut-off frequency and value of filter capacitor. $C_{f}$ is filter capacitor and $\omega_{n}$ is the nominal angular velocity of grid voltage.

The power control loop of PSC is sketched in Figure 9. This power controller only has an integrator, allowing a power mismatch between reference and power feedback. In fact, some articles point out the inherent mathematical equivalence between the droop control and swing equation [12][13].

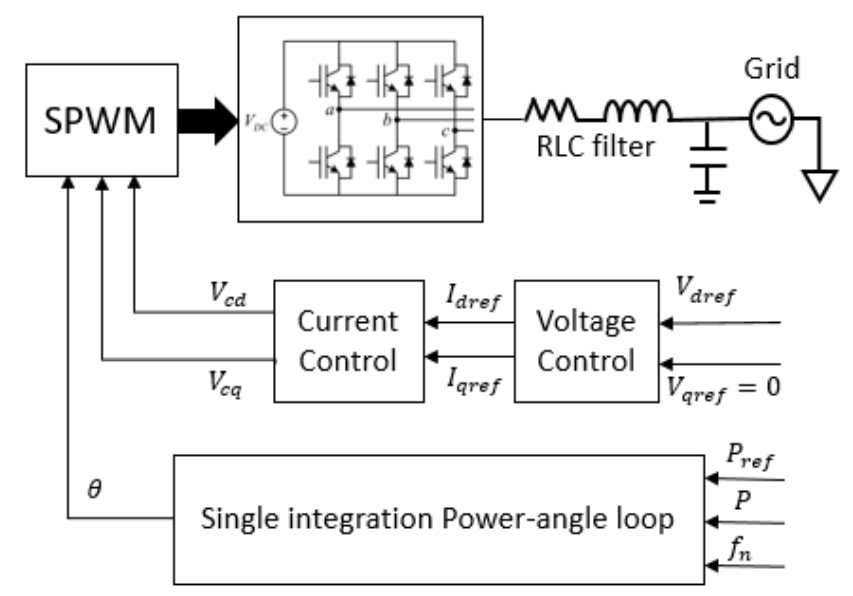

Figure 7 Power Synchronisation Control

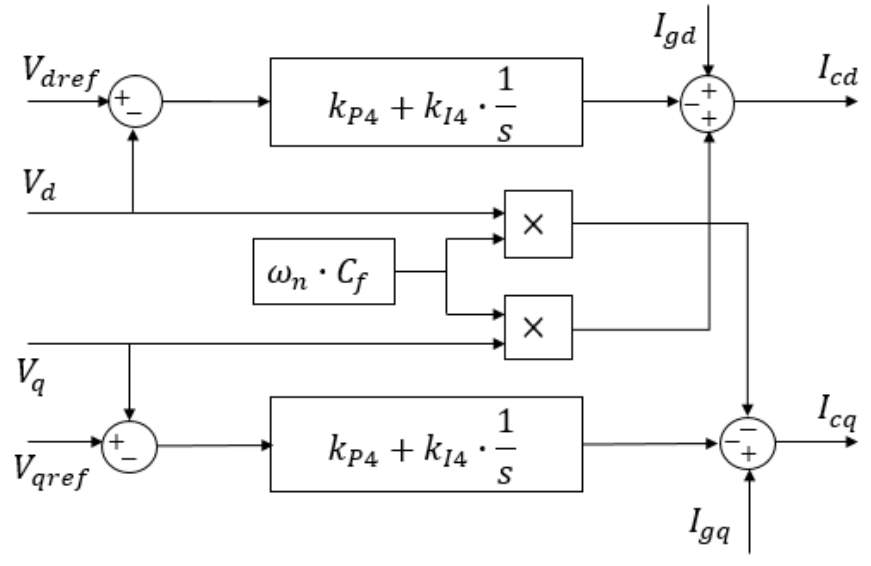

Figure 8 Voltage control

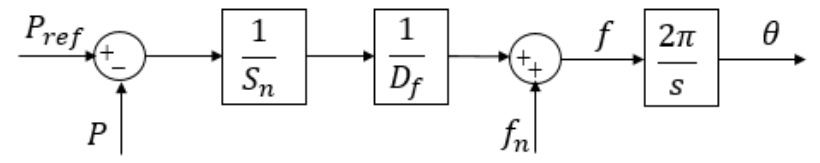

Figure 9 Single integration power control

\section{VSM testing and results}

In this section, the three controllers described in the previous section are compared and analysed under different situations including power reference tracking, phase step change, frequency step, and ramp change. The grid is assumed as three phase balanced voltage source with the frequency of $50 \mathrm{~Hz}$, line to line RMS voltage of $400 \mathrm{~V}$, and the converter has the nominal power up to $10 \mathrm{~kW}$ to emulate a wind turbine. To ensure the fairness of comparison, the power change and phase step change tests are designed to have similar dynamic performance for the three models when the controllers react to a change on power demand. The bandwidths of the inner and outer loops (in the CVC and PSC models) are kept separated by one decade. The bandwidth of current control loop is $200 \mathrm{~Hz}$ for both models, while the power and voltage control loops have the operating frequency range around $20 \mathrm{~Hz}$. The detailed parameter information for the grid and models can be seen in Table 1 and Table 2.

Table 1 Grid parameter information

\begin{tabular}{ll}
\hline Model name & Value \\
\hline Grid nominal setting & $V_{L L}=400 \mathrm{~V}$ \\
& $f_{n}=50 \mathrm{~Hz}$ \\
& $S_{n}=10 \mathrm{~kW}$ \\
\hline RLC filter & $R=0.16 \Omega$ \\
& $L=5.1 \times 10^{-3} \mathrm{H}$ \\
& $C_{f}=1 \times 10^{-6} \mathrm{~F}$ \\
\hline
\end{tabular}


Table 2 Model parameter information

\begin{tabular}{lll}
\hline Model name & PI controller & Other parameters \\
& parameters & \\
\hline CVC & $k_{P I}=1.0186$ & $k_{D}=1.2$ \\
& $k_{I I}=32$ & \\
& $k_{P 2 I}=0$ & \\
& $k_{I 21}=4.08 \times 10^{-2}$ & \\
& $k_{P 22}=0$ & \\
& $k_{I 22}=4.08 \times 10^{-2}$ & \\
& $k_{P 3}=100$ & $H=2.2 \mathrm{~s}$ \\
VSM & $k_{I 3}=1$ & $D=5 \times 10^{4}$ \\
\hline PSC & $k_{P 4}=4$ & $D_{f}=15$ \\
& $k_{I 4}=0.02$ & \\
\hline
\end{tabular}

\subsection{Power coherence test}

The power response to a reference step change is shown in Figure 10. At $0.2 \mathrm{~s}$, the converter power reference steps down by $3 \mathrm{~kW}$ starting at $5 \mathrm{~kW}$. At $0.7 \mathrm{~s}$, power reference steps up by $2 \mathrm{~kW}$, ending at $4 \mathrm{~kW}$.

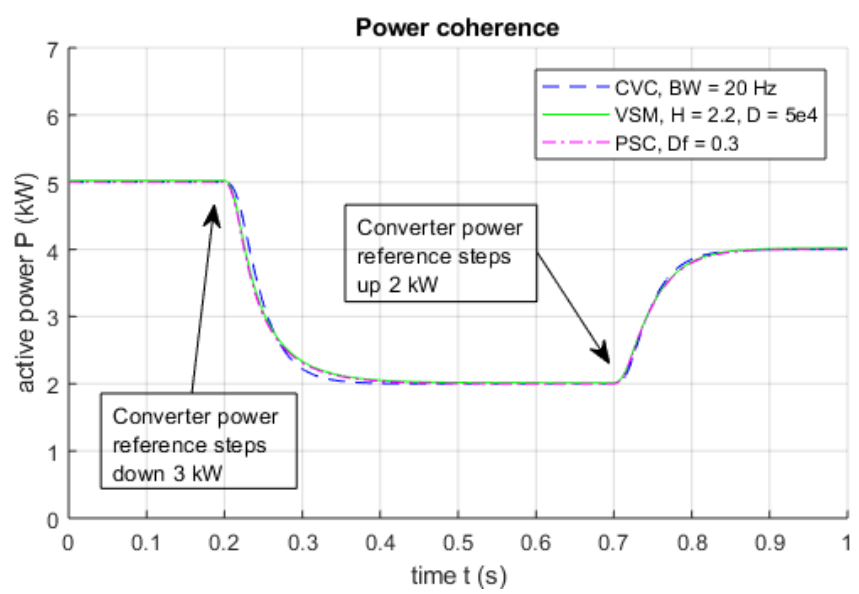

Figure 10 Power reference step test

Figure 10 shows that the 4 models own the same dynamic characteristic of the power. The four models are adjusted into the power coherence condition.

\subsection{Phase step change test}

Figure 11 shows the controllers' performance in response to different phase steps, $3^{\circ}, 9^{\circ}$ and $20^{\circ}$ respectively. The inertia emulation constant $k_{D}$ is set as 1.2. This ensures that the CVC model has similar behaviour to the VSM model when grid phase steps down by $3^{\circ}$.

The result shows that the VSM and PSC have similar behavior under the grid angle disturbance where the power increases immediately when the phase steps down, but PSC injects a little bit more power than the VSM. The power response between PSC and VSM usually has larger peak value than the converter nominal power and it is likely that this might trip the converter current protection.

For CVC, it is shown that adding the inertia emulation loop can make the converter response to the grid phase change, but $\mathrm{CVC}$ without the inertia emulation will not respond as shown in Figure 11. In addition, the response has two peaks during the disturbance, which is different from the VSM and PSC models and can have potentially detrimental effects on stability. Moreover, the increase in phase step does not affect the peak value of power response.

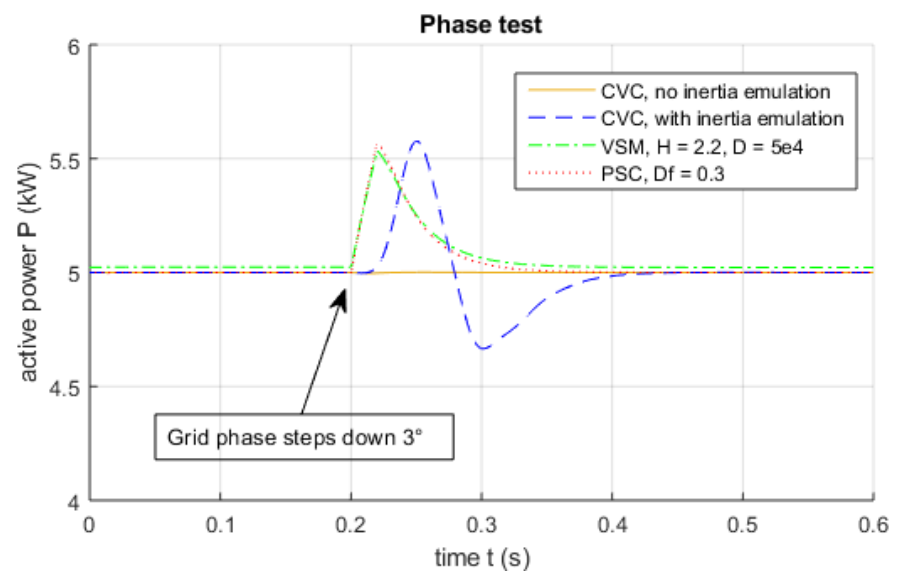

(a) Phase steps down by $3^{\circ}$

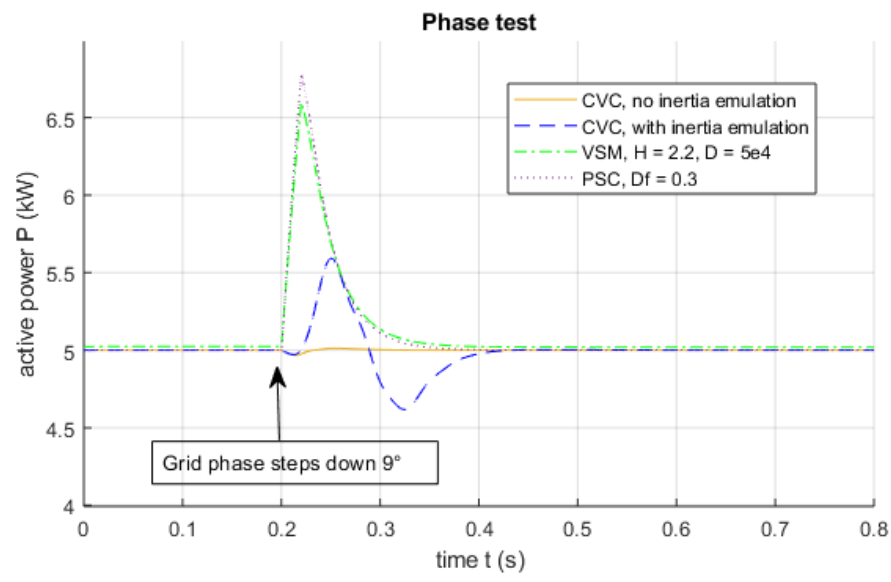

(b) Phase steps down by $9^{\circ}$

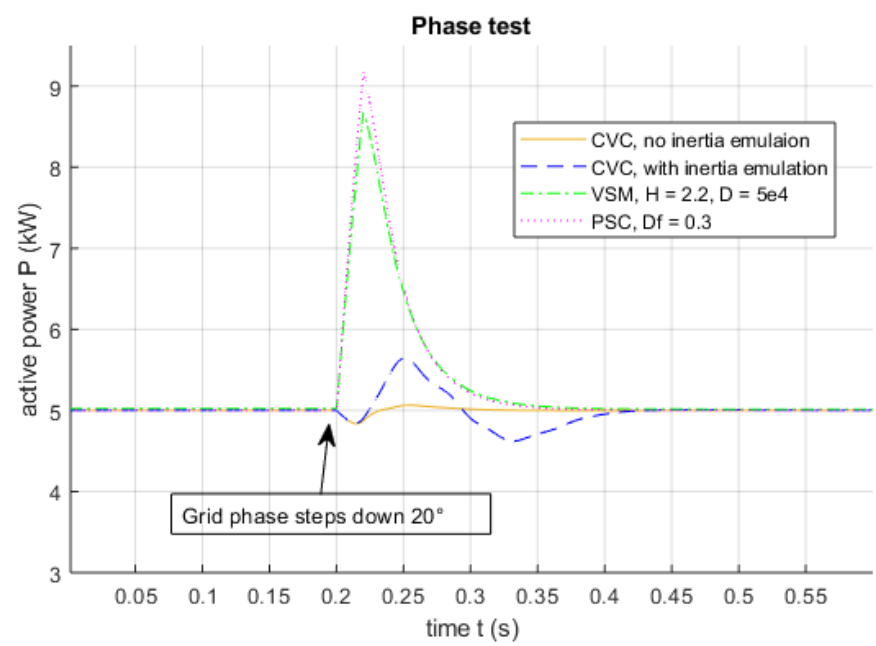

(c) Phase steps down by $20^{\circ}$

Figure 11 Grid phase step test for CVC without inertia emulation, CVC with inertia emulation, VSM and PSC models. 


\subsection{Frequency step change test}

The frequency step test is presented in Figure 12 where at $0.2 \mathrm{~s}$, grid frequency steps down by $1 \mathrm{~Hz}$.

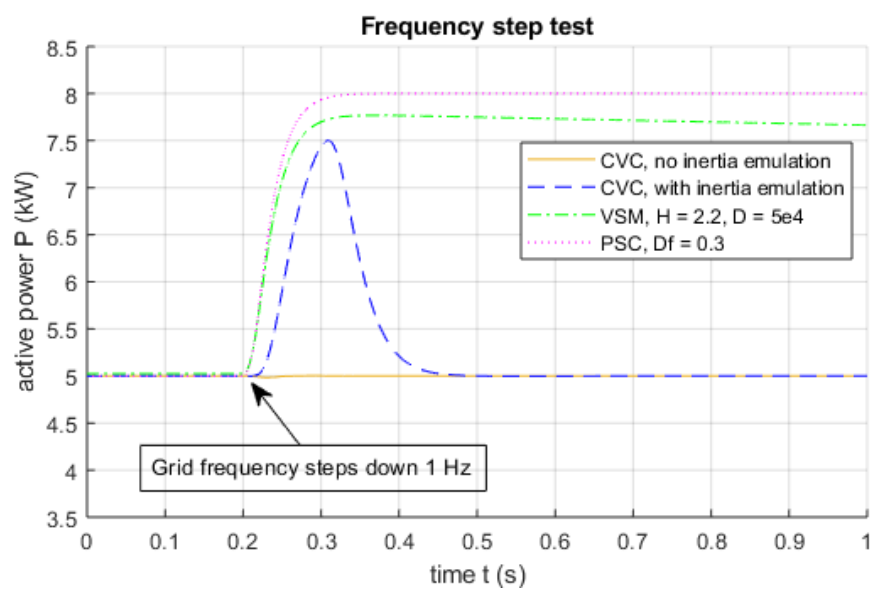

Figure 12 Frequency step test

The CVC without inertia emulation does not response to the grid frequency change. If the inertia emulation loop is added on $\mathrm{CVC}$, it shows a temporary power injection when grid frequency steps down. The power returns to reference value within $0.3 \mathrm{~s}$ after the disturbance as the value of ROCOF decreases to zero in steady state condition. The peak power of CVC is close to the VSM model, which might own to the power and phase coherence between the two models. PSC model has a continuous power injection due to the inherent droop effect. The power response of VSM can reach a high value at the beginning and then it will decrease to the reference power gradually as shown in Figure 13. The time for VSM to return to the reference depends on the setting of $H$ and $D$.

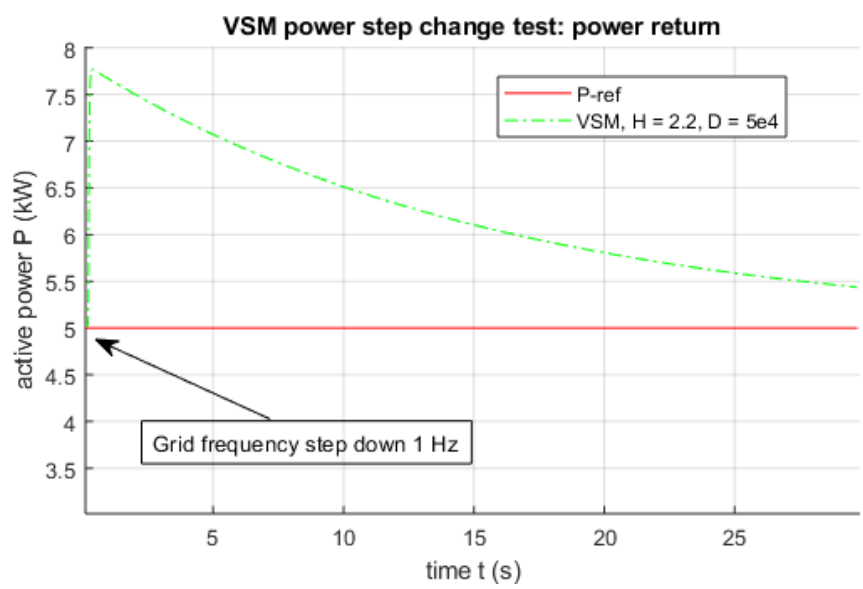

Figure 13 VSM power return test

\subsection{Frequency ramp change test}

The result of grid frequency ramp test is shown in Figure 14. At $0.2 \mathrm{~s}$, grid frequency ramps down with the rate of $1 \mathrm{~Hz} / \mathrm{s}$, and after 1 -second (at $1.2 \mathrm{~s}$ ) it stops ramping.

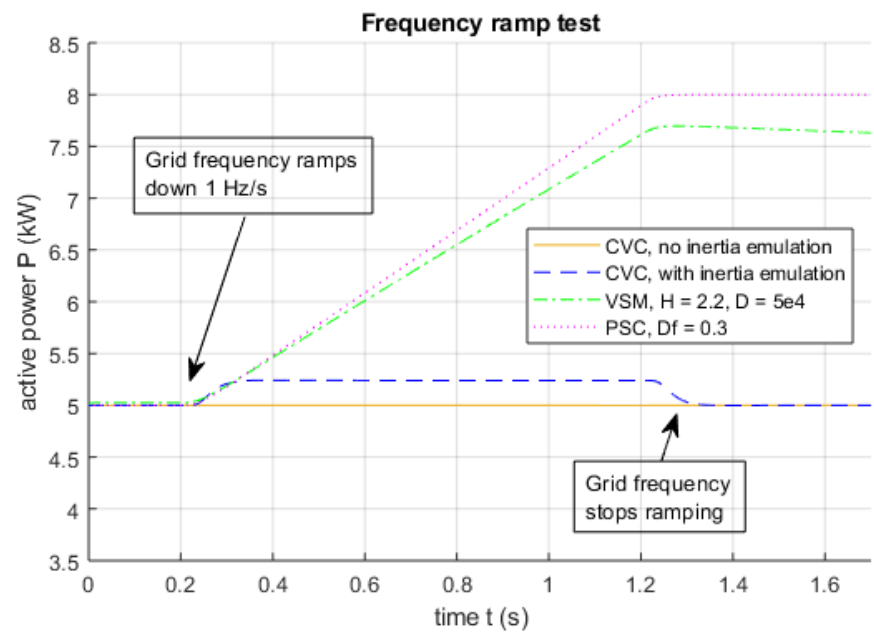

Figure 14 Frequency ramp test

For CVC model without inertia emulation, there is no power response. If inertia emulation loop is added, the CVC model will inject the constant power to the grid during the ramp period. It can be seen that both PSC and VSM models have a significant larger power injection to the grid than CVC. The VSM has less power injection than PSC due to the different inertia characteristic in configuration. When the frequency change stops ramping down, the power from CVC and VSM models will return to reference value but PSC keep injecting the power in a constant value due to its inherent droop effect.

\section{Conclusion}

The behaviour of three different models (CVC, VSM and PSC) under various types of disturbance are analysed and compared. When the power and phase response of the three models are maintained in the coherent condition, under the frequency response, $\mathrm{CVC}$ will provide $\mathrm{ROCOF}$ based inertial response, VSM will provide the temporary and large inertia response and the dynamic power behaviour depends on the $\mathrm{H}$ and $\mathrm{D}$ settings. PSC provides continuous and large power injection to the grid under frequency disturbance. All the three methods are able to provide the power support to more or less degree during the fault event, which verifies that the VSM can be a potential solution to enhance stable operation of conventional protection method.

\section{References}

[1] T, Ackerman., T, Prevost., V, Vittal., et al.: 'A future without inertia is closer than you think', IEEE power \& energy magazine., 2017

[2] A, Roscoe., M, Yu., A, Dyśko., et al,: 'A VSM ( Virtual Synchronous Machine ) Convertor Control Model Suitable for RMS Studies for Resolving System Operator/Owner Challenges', 2016, 15th Wind Integr. Work

[3] R, Li., C, D, Booth., A, Dysko., et al.: 'Development of models to study VSC response to AC system faults and the potential impact on network protection', IET UPEC Conference., 2014, pp. 1-6

[4] R, Li., J, Zhu., Q, Hong., et al.: 'Impact of low (zero) carbon power systems on power system protection: a new evaluation 
approach based on a flexible modelling and hardware testing platform', IET Renew. Power Gener., 2019, pp. 1-9

[5] V, Telukunta., J, Pradhan., A, Agrawal., et al.: 'Protection challenges under bulk penetration of renewable energy resources in power systems: A review', CSEE J. Power Energy Syst., 2017, 3, (4), pp. 365-379

[6] H, P, Beck. R, Hesse.: 'Virtual synchronous machine', 9th Int. Conf. Electr. Power Qual, 2007

[7] V, Converters., L, Zhang., L, Harnefors., et al.: 'PowerSynchronization Control of Grid-Connected Voltage-Source Converters', 2010, 25, (2), pp. 809-820

[8] L, R, Castillo., A, Roscoe., S, Member.: 'Experimental Stability Assessment of Converter-Dominated Electrical Grids', 16th Wind Integr. Work., 2017 pp. 1-6

[9] J, Morren., S, W, H, de Haan., W, L, Kling., et al.: 'Wind turbines emulating inertia and supporting primary frequency control', IEEE Trans. Power Syst., 2006, 21, (1), pp. 433-434 [10] Y, Amirnaser., R, Iravani.: 'VOLTAGE-SOURCED CONVERTERS IN POWER SYSTEMS. Modeling, Control, and Applications', (IEEE Press, 2001, 1st edn), pp. 204-244

[11] Z, Shuai., C, Shen., X, Yin., et al.: 'Fault Analysis of Inverter-Interfaced Distributed Generators with Different Control Schemes', IEEE Trans. Power Deliv., 2018, 33, (3), pp. 1223-1235

[12] S, D'Arco., J, A, Suul., 'Equivalence of virtual synchronous machines and frequency-droops for converterbased Microgrids,' IEEE Trans. Smart Grid, 2014, 5 (1), pp. 394-395

[13] S, D, Arco., J, A, Suul., 'Virtual Synchronous MachinesClassification of Implementations and Analysis of Equivalence to Droop Controllers for Microgrids.', 2013, IEEE Grenoble Conference. 